\title{
ON THE HYDROGEN ATOM VIA WIGNER-HEISENBERG ALGEBRA
}

\author{
R. de Lima Rodrigues \\ Unidade Acadêmica de Educação \\ Universidade Federal de Campina Grande, Cuité - PB, CEP 58.175-000- Brazil \\ Centro Brasileiro de Pesquisas Físicas (CBPF) \\ Rua Dr. Xavier Sigaud, 150, CEP 22290-180, Rio de Janeiro, RJ, Brazil
}

\begin{abstract}
We extend the usual Kustaanheimo-Stiefel $4 D \rightarrow 3 D$ mapping to study and discuss a constrained super-Wigner oscillator in four dimensions. We show that the physical hydrogen atom is the system that emerges in the bosonic sector of the mapped super $3 D$ system.
\end{abstract}

PACS numbers: 11.30.Pb, 03.65.Fd, 11.10.EF

E-mail to RLR is rafael@df.ufcg.edu.br or rafaelr@cbpf.br. J. Phys. A: Math. Theor. (2009) 42 355213. 


\section{INTRODUCTION}

The R-deformed Heisenberg or Wigner-Heisenberg (WH) algebraic technique [1] which was super-realized for quantum oscillators [2, 3, 4], is related to the paraboson relations introduced by Green [5].

Let us now point out that the WH algebra is given by following (anti-)commutation relations $\left([A, B]_{+} \equiv A B+B A\right.$ and $\left.[A, B]_{-} \equiv A B-B A\right)$ :

$$
H=\frac{1}{2}\left[a^{-}, a^{+}\right]_{+}, \quad\left[H, a^{ \pm}\right]_{-}= \pm a^{ \pm}, \quad\left[a^{-}, a^{+}\right]_{-}=1+c R,
$$

where $c$ is a real constant associated to the Wigner parameter [2] and the $R$ operator satisfies

$$
\left[R, a^{ \pm}\right]_{+}=0, \quad R^{2}=1
$$

Note that when $c=0$ we have the standard Heisenberg algebra.

The generalized quantum condition given in Eq. (11) has been found relevant in the context of integrable models [6]. Furthermore, this algebra was also used to solve the energy eigenvalue and eigenfunctions of the Calogero interaction, in the context of one-dimensional many-body integrable systems, in terms of a new set of phase space variables involving exchanged operators [7, 8]. From this WH algebra a new kind of deformed calculus has been developed [9, 10, 11].

The WH algebra has been considered for the three-dimensional non-canonical oscillator to generate a representation of the orthosympletic Lie superalgebra $\operatorname{osp}(3 / 2)$ [12], and recently Palev et. al. have investigated the $3 D$ Wigner oscillator under a discrete non-commutative context [13, 14]. Also, the connection of the WH algebra with the Lie superalgebra $s \ell(1 \mid n)$ has been studied in a detailed manner [15].

Recently, the relevance of relations (11) to quantization in fractional dimension has been also discussed [16, 17] and the properties of Weyl-ordered polynomials in operators $P$ and $Q$, in fractional-dimensional quantum mechanics have been developed [18].

The Kustaanheimo-Stiefel mapping [19] yields the Schrödinger equation for the hydrogen atom that has been exactly solved and well-studied in the literature. (See for example, Chen [20], Cornish [21], Chen and Kibler [22], D'Hoker and Vinet [23].) Kostelecky, Nieto and Truax [24] have studied in a detailed manner the relation of the supersymmetric (SUSY) 
Coulombian problem [25, 26, 27, 28, 29] in D-dimensions with that of SUSY isotropic oscillators in D-dimensions in the radial version. (See also Lahiri et. al. [30]) For the mapping with $3 D$ radial oscillators, see also Bergmann and Frishman [31], Cahill [32] and J. - L. Chen et. al. [33].

The connection of the D-dimensional hydrogen atom with the D-dimensional harmonic oscillator in terms of the $s u(1,1)$ algebra has been investigated by Gao-Jian Zeng et. al. [34]. However, the correspondence mapping of a $4 D$ isotropic constrained super Wigner oscillator (for super Wigner oscillators see our previews work [2, 3]) with the corresponding super system in $3 D$ such that the usual $3 D$ hydrogen atom emerges in the $4 D \rightarrow 3 D$ mapping in the bosonic sector has not been studied in the literature; the objectives of the present work are to do such a mapping and to analyze in detail the consequences. In this work, the stationary states of the hydrogen atom are mapped onto the super-Wigner oscillator by using the Kustaanheimo-Stiefel transformation.

This work is organized as follows. In Section II, we start by summarizing the R-deformed Heisenberg algebra or Wigner-Heisenberg algebraic technique for the Wigner oscillator, based on the super-realization of the WH algebra for simpler effective spectral resolutions

of general oscillator-related potentials, applied by Jayaraman and Rodrigues, in Ref. [2]. In Section III, we illustrate how to construct the $4 D \rightarrow 3 D$ mapping in the bosonic sector which offer's a simple resolution of the hydrogen energy spectra and eigenfunctions. The conclusion is given in Section IV.

\section{THE SUPER WIGNER OSCILLATOR IN 1D}

The Wigner oscillator ladder operators

$$
a^{ \pm}=\frac{1}{\sqrt{2}}\left( \pm i \hat{p}_{x}-\hat{x}\right)
$$

of the WH algebra may be written in terms of the super-realization of the position and momentum operators viz., $\hat{x}=x \Sigma_{1}$ and $\hat{p}_{x}=-i \Sigma_{1} \frac{d}{d x}+\frac{c}{2 x} \Sigma_{2}$, satisfy the general quantum rule $\left[\hat{x}, \hat{p}_{x}\right]_{-}=i(1+c R)$, where $c=2(\ell+1)$. Thus, in this representation the reflection operator becomes $R=\Sigma_{3}$, where $\Sigma_{3}$ is the diagonal Pauli matrix.

Thus, from the super-realized first order ladder operators given by 


$$
a^{ \pm}(\ell+1)=\frac{1}{\sqrt{2}}\left\{ \pm \frac{d}{d x} \pm \frac{(\ell+1)}{x} \Sigma_{3}-x\right\} \Sigma_{1}, \quad \ell>0
$$

the Wigner Hamiltonian becomes

$$
H(\ell+1)=\frac{1}{2}\left[a^{+}(\ell+1), a^{-}(\ell+1)\right]_{+}
$$

and the WH algebra ladder relations are readily obtained as

$$
\left[H(\ell+1), a^{ \pm}(\ell+1)\right]_{-}= \pm a^{ \pm}(\ell+1) .
$$

Equations (5) and (6) together with the commutation relation

$$
\left[a^{-}(\ell+1), a^{+}(\ell+1)\right]_{-}=1+2(\ell+1) \Sigma_{3}
$$

constitute the super WH algebra.

Thus, the super Wigner oscillator Hamiltonian in terms of the Pauli's matrices $\left(\Sigma_{i}\right.$, $\mathrm{i}=1,2,3)$ is given by

$$
\begin{aligned}
H(\ell+1) & =\frac{1}{2}\left\{-\frac{d^{2}}{d x^{2}}+x^{2}+\frac{1}{x^{2}}(\ell+1)\left[(\ell+1) \Sigma_{3}-1\right] \Sigma_{3}\right\} \\
& =\left(\begin{array}{cc}
H_{-}(\ell) & 0 \\
0 & H_{+}(\ell)=H_{-}(\ell+1)
\end{array}\right)
\end{aligned}
$$

where the bosonic and fermionic sector Hamiltonians are respectively given by

$$
H_{-}(\ell)=\frac{1}{2}\left\{-\frac{d^{2}}{d x^{2}}+x^{2}+\frac{1}{x^{2}} \ell(\ell+1)\right\}
$$

and

$$
H_{+}(\ell)=\frac{1}{2}\left\{-\frac{d^{2}}{d x^{2}}+x^{2}+\frac{1}{x^{2}}(\ell+1)(\ell+2)\right\}=H_{-}(\ell+1) .
$$

Note that the bosonic sector is the Hamiltonian of the oscillator with barrier.

The super Wigner oscillator eigenfunctions that generate the eigenspace associated with even(odd) $\Sigma_{3}$-parity for bosonic(fermionic) quanta $n=2 m(n=2 m+1)$ are given by

$$
\Psi_{n=2 m}(x ; \ell+1)=\left(\begin{array}{c}
\psi_{-}^{(m)}(x ; \ell) \\
0
\end{array}\right), \quad \Psi_{n=2 m+1}(x ; \ell+1)=\left(\begin{array}{c}
0 \\
\psi_{+}^{(m)}(x ; \ell)
\end{array}\right)
$$


and satisfy the following eigenvalue equation

$$
\begin{aligned}
H(\ell+1) \Psi_{n}(x ; \ell+1) & =E_{n} \Psi_{n}(x ; \ell+1) \\
\Sigma_{3} \Psi_{n=2 m} & =\Psi_{n=2 m} \\
\Sigma_{3} \Psi_{n=2 m+1} & =-\Psi_{n=2 m+1}
\end{aligned}
$$

where the non-degenerate energy eigenvalues are obtained by the repeated application of the raising operator on the ground eigenstate

$$
\Psi_{n}(x ; \ell+1) \propto\left(a^{+}(\ell+1)\right)^{n} \Psi_{0}(x ; \ell+1)
$$

and are given by

$$
E_{n}=\ell+\frac{3}{2}+n, \quad n=0,1,2, \ldots
$$

The ground state energy eigenfunction satisfies the following annihilation condition

$$
a^{-}(\ell+1) \Psi_{(0)}(x ; \ell+1)=0
$$

which using Eq. (4) result in

$$
\psi_{-}^{(0)}(x ; \ell)=N_{1} x^{(\ell+1)} e^{-\frac{x^{2}}{2}}, \quad \psi_{+}^{(0)}(x ; \ell)=N_{2} x^{-(\ell+1)} e^{-\frac{x^{2}}{2}} .
$$

If we assume $\ell+1>0$, only $\psi_{-}^{(0)}(x ; \ell)$ meets the physical requirement of vanishing at the origin and $\psi_{+}^{(0)}(x ; \ell)$, which does not stand this test, is discard by setting $N_{2}=0$ in (15).

In this case, the normalizable ground-state eigenfunction is given, up to a normalization constant, by

$$
\Psi_{0}(x ; \ell+1) \propto\left(\begin{array}{c}
x^{(\ell+1)} e^{-\frac{1}{2} x^{2}} \\
0
\end{array}\right),
$$

which has even $\Sigma_{3}$-parity, i.e. $\Sigma_{3} \Psi_{0}(x ; \ell+1)=\Psi_{0}(x ; \ell+1)$.

For the bosonic and fermionic sector Hamiltonians the energy eigenvectors satisfy the following equations

$$
H_{ \pm}(\ell) \psi_{ \pm}^{(m)}(x ; \ell)=E_{ \pm}^{(m)} \psi_{ \pm}^{(m)}(x ; \ell)
$$


where the eigenvalues are exactly constructed via WH algebra ladder relations and are given by

$$
E_{-}^{(m)}=E_{0}+2 m, \quad E_{+}^{(m)}=E_{0}+2(m+1), \quad m=0,1,2, \ldots,
$$

where $E_{0}$ is the energy of the Wigner oscillator ground state. Note that the energy spectrum of a particle in a potential given by bosonic sector Hamiltonian is equally spaced like that of the 3D isotropic harmonic oscillator, with difference of two quanta between two levels.

Also, note that the operators $a^{ \pm}(\ell+1)$ given in Eq. (44) together with $H(\ell+1), J_{ \pm}=$ $\left(a^{ \pm}(\ell+1)\right)^{2}$ satisfy an $\operatorname{osp}(1 \mid 2)$ superalgebra.

\section{THE CONSTRAINED SUPER WIGNER OSCILLATOR IN $4 D$}

The usual isotropic oscillator in $4 D$ has the following eigenvalue equation for it's Hamiltonian $H_{\mathrm{OSc}}^{B}$, described by (employing natural system of units $\hbar=m=1$ ) time-independent Schrödinger equation

$$
H_{o s c}^{\mathrm{B}} \Psi_{o s c}^{\mathrm{B}}(y)=E_{o s c}^{\mathrm{B}} \Psi_{o s c}^{\mathrm{B}}(y)
$$

with

$$
\begin{gathered}
H_{\text {osc }}^{\mathrm{B}}=-\frac{1}{2} \nabla_{4}^{2}+\frac{1}{2} s^{2}, \quad s^{2}=\Sigma_{i=1}^{4} y_{i}^{2}, \\
\nabla_{4}^{2}=\frac{\partial^{2}}{\partial y_{1}^{2}}+\frac{\partial^{2}}{\partial y_{2}^{2}}+\frac{\partial^{2}}{\partial y_{3}^{2}}+\frac{\partial^{2}}{\partial y_{4}^{2}}=\sum_{i=1}^{4} \frac{\partial^{2}}{\partial y_{i}^{2}},
\end{gathered}
$$

where the superscript $B$ in $H_{\mathrm{OSC}}^{B}$ is in anticipation of the Hamiltonian, with constraint to be defined, being implemented in the bosonic sector of the super $4 D$ Wigner system with unitary frequency. Changing to spherical coordinates in 4-space dimensions, allowing a factorization of the energy eigenfunctions as a product of a radial eigenfunction and spinspherical harmonic.

In (21), the coordinates $y_{i}(i=1,2,3,4)$ in spherical coordinates in $4 \mathrm{D}$ are defined by [20, 23] 


$$
\begin{aligned}
& y_{1}=s \cos \left(\frac{\theta}{2}\right) \cos \left(\frac{\varphi-\omega}{2}\right) \\
& y_{2}=s \cos \left(\frac{\theta}{2}\right) \sin \left(\frac{\varphi-\omega}{2}\right) \\
& y_{3}=s \sin \left(\frac{\theta}{2}\right) \cos \left(\frac{\varphi+\omega}{2}\right) \\
& y_{4}=s \sin \left(\frac{\theta}{2}\right) \sin \left(\frac{\varphi+\omega}{2}\right),
\end{aligned}
$$

where $0 \leq \theta \leq \pi, \quad 0 \leq \varphi \leq 2 \pi$ and $0 \leq \omega \leq 4 \pi$.

The mapping of the coordinates $y_{i}(i=1,2,3,4)$ in $4 D$ with the Cartesian coordinates $\rho_{i}(i=1,2,3)$ in $3 D$ is given by the Kustaanheimo-Stiefel transformation

$$
\begin{aligned}
& \rho_{i}=\sum_{a, b=1}^{2} z_{a}^{*} \Gamma_{a b}^{i} z_{b}, \quad(i=1,2,3) \\
& z_{1}=y_{1}+i y_{2}, \quad z_{2}=y_{3}+i y_{4}
\end{aligned}
$$

where the $\Gamma_{a b}^{i}$ are the elements of the usual Pauli matrices. If one defines $z_{1}$ and $z_{2}$ as in Eq. (24), $Z=\left(\begin{array}{l}z_{1} \\ z_{2}\end{array}\right)$ is a two dimensional spinor of $S U(2)$ transforming as $Z \rightarrow Z^{\prime}=U Z$ with $U$ a two-by-two matrix of $S U(2)$ and of course $Z^{\dagger} Z$ is invariant. So the transformation (23) is very spinorial. Also, using the standard Euler angles parametrizing $S U(2)$ as in transformations (22) and (24) one obtains

$$
\begin{aligned}
& z_{1}=s \cos \left(\frac{\theta}{2}\right) e^{\frac{i}{2}(\varphi-\omega)} \\
& z_{2}=s \sin \left(\frac{\theta}{2}\right) e^{\frac{i}{2}(\varphi+\omega)} .
\end{aligned}
$$

Note that the angles in these equations are divided by two. However, in 3D, the angles are not divided by two, viz., $\rho_{3}=\rho \cos ^{2}\left(\frac{\theta}{2}\right)-\rho \sin ^{2}\left(\frac{\theta}{2}\right)=\rho \cos \theta$. Indeed, from (23) and (25), we obtain

$$
\rho_{1}=\rho \sin \theta \cos \varphi, \quad \rho_{2}=\rho \sin \theta \sin \varphi, \quad \rho_{3}=\rho \cos \theta
$$

and also that 


$$
\begin{aligned}
\rho & =\left\{\rho_{1}^{2}+\rho_{2}^{2}+\rho_{3}^{2}\right\}^{\frac{1}{2}}=\left\{\left(\rho_{1}+i \rho_{2}\right)\left(\rho_{1}-i \rho_{2}\right)+\rho_{3}^{2}\right\}^{\frac{1}{2}} \\
& =\left\{\left(2 z_{1}^{*} z_{2}\right)\left(2 z_{1} z_{2}^{*}\right)+\left(z_{1}^{*} z_{1}-z_{2}^{*} z_{2}\right)^{2}\right\}^{\frac{1}{2}} \\
& =\left(z_{1} z_{1}^{*}+z_{2} z_{2}^{*}\right)=\sum_{i=1}^{4} y_{i}^{2}=s^{2} .
\end{aligned}
$$

The complex form of the Kustaanheimo-Stiefel transformation was given by Cornish [21].

Thus, the expression for $H_{o s c}^{\mathrm{B}}$ in (20) can be written in the form

$$
\begin{aligned}
H_{o s c}^{\mathrm{B}} & =-\frac{1}{2}\left(\frac{\partial^{2}}{\partial s^{2}}+\frac{3}{s} \frac{\partial}{\partial s}\right) \\
& -\frac{2}{s^{2}}\left[\frac{1}{\sin \theta} \frac{\partial}{\partial \theta} \sin \theta \frac{\partial}{\partial \theta}+\frac{1}{\sin ^{2} \theta} \frac{\partial^{2}}{\partial \varphi^{2}}+\frac{1}{\sin ^{2} \theta}\left(2 \cos \theta \frac{\partial}{\partial \varphi}+\frac{\partial}{\partial \omega}\right) \frac{\partial}{\partial \omega}\right]+\frac{1}{2} s^{2} .
\end{aligned}
$$

We obtain a constraint by projection (or "dimensional reduction") from four to three dimensional. Note that $\psi_{\text {osc }}^{\mathrm{B}}$ is independent of $\omega$ provides the constraint condition

$$
\frac{\partial}{\partial \omega} \Psi_{o s c}^{\mathrm{B}}(s, \theta, \varphi)=0
$$

imposed on $H_{\text {osc }}^{\mathrm{B}}$, the expression for this restricted Hamiltonian, which we continue to call as $H_{o s c}^{\mathrm{B}}$, becomes

$$
H_{\text {osc }}^{\mathrm{B}}=-\frac{1}{2}\left(\frac{\partial^{2}}{\partial s^{2}}+\frac{3}{s} \frac{\partial}{\partial s}\right)-\frac{2}{s^{2}}\left[\frac{1}{\sin \theta} \frac{\partial}{\partial \theta} \sin \theta \frac{\partial}{\partial \theta}+\frac{1}{\sin ^{2} \theta} \frac{\partial^{2}}{\partial \varphi^{2}}\right]+\frac{1}{2} s^{2} .
$$

Identifying the expression in bracket in (30) with $L^{2}$, the square of the orbital angular momentum operator in $3 D$, since we always have

$$
L^{2}=(\vec{\sigma} \cdot \vec{L})(\vec{\sigma} \cdot \vec{L}+1)
$$

which is valid for any system, where $\sigma_{i}(i=1,2,3)$ are the Pauli matrices representing the spin $\frac{1}{2}$ degrees of freedom, we obtain for $H_{o s c}^{\mathrm{B}}$ the final expression

$$
H_{o s c}^{\mathrm{B}}=\frac{1}{2}\left[-\left(\frac{\partial^{2}}{\partial s^{2}}+\frac{3}{s} \frac{\partial}{\partial s}\right)+\frac{4}{s^{2}}(\vec{\sigma} \cdot \vec{L})(\vec{\sigma} \cdot \vec{L}+1)+s^{2}\right] .
$$


Now, associating $H_{o s c}^{\mathrm{B}}$ with the bosonic sector of the super Wigner system, $H_{\mathrm{W}}$, subject to the same constraint as in (29), and following the analogy with the Section II of construction of super Wigner systems, we first must solve the Schrödinger equation

$$
H_{\mathrm{W}} \Psi_{\mathrm{W}}(s, \theta, \varphi)=E_{\mathrm{W}} \Psi_{\mathrm{W}}(s, \theta, \varphi),
$$

where the explicit form of $H_{\mathrm{w}}$ is given by

$$
\begin{aligned}
& H_{\mathrm{W}}\left(2 \vec{\sigma} \cdot \vec{L}+\frac{3}{2}\right)= \\
& \left(\begin{array}{cc}
-\frac{1}{2}\left(\frac{\partial}{\partial s}+\frac{3}{2 s}\right)^{2}+\frac{1}{2} s^{2}+\frac{\left(2 \vec{\sigma} \cdot \vec{L}+\frac{1}{2}\right)\left(2 \vec{\sigma} \cdot \vec{L}+\frac{3}{2}\right)}{2 s^{2}} & 0 \\
0 & -\frac{1}{2}\left(\frac{\partial}{\partial s}+\frac{3}{2 s}\right)^{2}+\frac{1}{2} s^{2}+\frac{\left(2 \vec{\sigma} \cdot \vec{L}+\frac{3}{2}\right)\left(2 \vec{\sigma} \cdot \vec{L}+\frac{5}{2}\right)}{2 s^{2}}
\end{array}\right) \cdot(34
\end{aligned}
$$

Using the operator technique in references [2, 3], we begin with the following superrealized mutually adjoint operators

$$
a_{\mathrm{W}}^{ \pm} \equiv a^{ \pm}\left(2 \vec{\sigma} \cdot \vec{L}+\frac{3}{2}\right)=\frac{1}{\sqrt{2}}\left[ \pm\left(\frac{\partial}{\partial s}+\frac{3}{2 s}\right) \Sigma_{1} \mp \frac{1}{s}\left(2 \vec{\sigma} \cdot \vec{L}+\frac{3}{2}\right) \Sigma_{1} \Sigma_{3}-\Sigma_{1} s\right]
$$

where $\vec{\Sigma}_{i}(i=1,2,3)$ constitute a set of Pauli matrices that provide the fermionic coordinates commuting with the similar Pauli set $\sigma_{i}(i=1,2,3)$ already introduced representing the spin $\frac{1}{2}$ degrees of freedom.

It is checked, after some algebra, that $a^{+}$and $a^{-}$of (35) are indeed the raising and lowering operators for the spectra of the super Wigner Hamiltonian $H_{\mathrm{W}}$ and they satisfy the following (anti-)commutation relations of the WH algebra:

$$
\begin{aligned}
& H_{\mathrm{W}}=\frac{1}{2}\left[a_{\mathrm{W}}^{-}, a_{\mathrm{W}}^{+}\right]_{+} \\
& =a_{\mathrm{W}}^{+} a_{\mathrm{W}}^{-}+\frac{1}{2}\left[1+2\left(2 \vec{\sigma} \cdot \vec{L}+\frac{3}{2}\right) \Sigma_{3}\right] \\
& =a_{\mathrm{W}}^{-} a_{\mathrm{W}}^{+}-\frac{1}{2}\left[1+2\left(2 \vec{\sigma} \cdot \vec{L}+\frac{3}{2}\right) \Sigma_{3}\right] \\
& {\left[H_{\mathrm{W}}, a_{\mathrm{W}}^{ \pm}\right]_{-}= \pm a_{\mathrm{W}}^{ \pm}} \\
& {\left[a_{\mathrm{W}}^{-}, a_{\mathrm{W}}^{+}\right]_{-}=1+2\left(2 \vec{\sigma} \cdot \vec{L}+\frac{3}{2}\right) \Sigma_{3},}
\end{aligned}
$$




$$
\left[\Sigma_{3}, a_{\mathrm{W}}^{ \pm}\right]_{+}=0 \Rightarrow\left[\Sigma_{3}, H_{\mathrm{W}}\right]_{-}=0 .
$$

Since the operator $\left(2 \vec{\sigma} \cdot \vec{L}+\frac{3}{2}\right)$ commutes with the basic elements $a^{ \pm}, \Sigma_{3}$ and $H_{\mathrm{W}}$ of the WH algebra (36), (37) and (38) it can be replaced by its eigenvalues $\left(2 \ell+\frac{3}{2}\right)$ and $-\left(2 \ell+\frac{5}{2}\right)$ while acting on the respective eigenspace in the from

$$
\Psi_{\mathrm{OSC}}(s, \theta, \varphi)=\left(\begin{array}{c}
\Psi_{\mathrm{OSC}}^{\mathrm{B}}(s, \theta, \varphi) \\
\Psi_{\mathrm{OSC}}^{\mathrm{F}}(s, \theta, \varphi)
\end{array}\right)=\left(\begin{array}{c}
R_{\mathrm{OSC}}^{\mathrm{B}}(s) \\
R_{\mathrm{OSC}}^{\mathrm{F}}(s)
\end{array}\right) y_{ \pm}(\theta, \varphi)
$$

in the notation where $y_{ \pm}(\theta, \varphi)$ are the spin-spherical harmonics [35, 36],

$$
\begin{aligned}
& y_{+}(\theta, \varphi)=y_{\ell \frac{1}{2} ; j=\ell+\frac{1}{2}, m_{j}}(\theta, \varphi) \\
& y_{-}(\theta, \varphi)=y_{\ell+1 \frac{1}{2} ; j=(\ell+1)-\frac{1}{2}, m_{j}}(\theta, \varphi)
\end{aligned}
$$

so that, we obtain: $(\vec{\sigma} \cdot \vec{L}+1) y_{ \pm}= \pm(\ell+1) y_{ \pm}, \quad\left(2 \vec{\sigma} \cdot \vec{L}+\frac{3}{2}\right) y_{+}=\left(2 \ell+\frac{3}{2}\right) y_{+}$and $\left(2 \vec{\sigma} \cdot \vec{L}+\frac{3}{2}\right) y_{-}=$ $-\left[2(\ell+1)+\frac{1}{2}\right] y_{-}$. Note that on these subspaces the 3D WH algebra is reduced to a formal $1 \mathrm{D}$ radial form with $H_{\mathrm{W}}\left(2 \vec{\sigma} \cdot \vec{L}+\frac{3}{2}\right)$ acquiring respectively the forms $H_{\mathrm{W}}\left(2 \ell+\frac{3}{2}\right)$ and

$$
H_{\mathrm{W}}\left(-2 \ell-\frac{5}{2}\right)=\Sigma_{1} H_{\mathrm{W}}\left(2 \ell+\frac{3}{2}\right) \Sigma_{1}
$$

Thus, the positive finite form of $H_{\mathrm{W}}$ in (36) together with the ladder relations (37) and the form (38) leads to the direct determination of the state energies and the corresponding Wigner ground state wave functions by the simple application of the annihilation conditions

$$
a^{-}\left(2 \ell+\frac{3}{2}\right)\left(\begin{array}{c}
R_{\mathrm{OSC}}^{B^{(0)}}(s) \\
R_{\mathrm{OSC}}^{F^{(0)}}(s)
\end{array}\right)=0 .
$$

Then, the complete energy spectrum for $H_{\mathrm{W}}$ and the whole set of energy eigenfunctions $\Psi_{\mathrm{OSC}}^{(n)}(s, \theta, \varphi)(n=2 m, 2 m+1, m=0,2 \cdots)$ follows from the step up operation provided by $a^{+}\left(2 \ell+\frac{3}{2}\right)$ acting on the ground state, which are also simultaneous eigenfunctions of the fermion number operator $N=\frac{1}{2}\left(1-\Sigma_{3}\right)$. We obtain for the bosonic sector Hamiltonian $H_{\mathrm{OSc}}^{B}$ with fermion number $n_{f}=0$ and even orbital angular momentum $\ell_{4}=2 \ell,(\ell=0,1,2 \ldots)$, the complete energy spectrum and eigenfunctions given by

$$
\left[E_{\text {osc }}^{B}\right]_{\ell_{4}=2 \ell}^{(m)}=2 \ell+2+2 m, \quad(m=0,1,2, \ldots),
$$




$$
\left[\Psi_{\text {osc }}^{B}(s, \theta, \varphi)\right]_{\ell_{4}=2 \ell}^{(m)} \propto s^{2 \ell} \exp \left(-\frac{1}{2} s^{2}\right) L_{m}^{(2 \ell+1)}\left(s^{2}\right)\left\{\begin{array}{l}
y_{+}(\theta, \varphi) \\
y_{-}(\theta, \varphi)
\end{array}\right.
$$

where $L_{m}^{\alpha}\left(s^{2}\right)$ are generalized Laguerre polynomials [2]. Now, to relate the mapping of the $4 \mathrm{D}$ super Wigner system given by (86) with the corresponding system in 3D, we make use of the substitution of $s^{2}=\rho$, Eq. (29) and the following substitutions

$$
\frac{\partial}{\partial s}=2 \sqrt{\rho} \frac{\partial}{\partial \rho}, \quad \frac{\partial^{2}}{\partial s^{2}}=4 \rho \frac{\partial^{2}}{\partial \rho^{2}}+2 \frac{\partial}{\partial \rho},
$$

in (34) and divide the eigenvalue equation for $H_{\mathrm{W}}$ in (33) by $4 s^{2}=4 \rho$, obtaining

$$
\begin{aligned}
& \left(\begin{array}{cc}
-\frac{1}{2}\left(\frac{\partial^{2}}{\partial \rho^{2}}+\frac{2}{\rho} \frac{\partial}{\partial \rho}\right)-\frac{1}{2}\left[-\frac{1}{4}-\frac{\vec{\sigma} \cdot \vec{L}(\vec{\sigma} \cdot \vec{L}+1)}{\rho^{2}}\right] & 0 \\
0 & -\frac{1}{2}\left(\frac{\partial^{2}}{\partial \rho^{2}}+\frac{2}{\rho} \frac{\partial}{\partial \rho}\right)-\frac{1}{2}\left[-\frac{1}{4}-\frac{\left(\vec{\sigma} \cdot \vec{L}+\frac{1}{2}\right)\left(\vec{\sigma} \cdot \vec{L}+\frac{3}{2}\right)}{\rho^{2}}\right]
\end{array}\right)\left(\begin{array}{l}
\Psi^{B} \\
\Psi^{F}
\end{array}\right) \\
& =\frac{1}{4 \rho} E_{\mathrm{W}}\left(\begin{array}{l}
\Psi^{B} \\
\Psi^{F}
\end{array}\right) .
\end{aligned}
$$

The bosonic sector of the above eigenvalue equation can immediately be identified with the eigenvalue equation for the Hamiltonian of the 3D Hydrogen-like atom expressed in the equivalent form given by

$$
\left\{-\frac{1}{2}\left(\frac{\partial^{2}}{\partial \rho^{2}}+\frac{2}{\rho} \frac{\partial}{\partial \rho}\right)-\frac{1}{2}\left[-\frac{1}{4}-\frac{\vec{\sigma} \cdot \vec{L}(\vec{\sigma} \cdot \vec{L}+1)}{\rho^{2}}\right]\right\} \psi(\rho, \theta, \varphi)=\frac{\lambda}{2 \rho} \psi(\rho, \theta, \varphi),
$$

where $\Psi^{B}=\psi(\rho, \theta, \varphi)$ and the connection between the dimensionless and dimensionfull eigenvalues, respectively, $\lambda$ and $E_{a}$ with $e=1=m=\hbar$ is given by [36]

$$
\lambda=\frac{Z}{\sqrt{-2 E_{a}}}, \quad \rho=\alpha r, \quad \alpha=\sqrt{-8 E_{a}},
$$

where $E_{a}$ is the energy of the electron Hydrogen-like atom, $(r, \theta, \varphi)$ stand for the spherical polar coordinates of the position vector $\vec{r}=\left(x_{1}, x_{2}, x_{3}\right)$ of the electron in relative to the nucleons of charge $Z$ together with $s^{2}=\rho$. We see then from equations (44), (45), (48) and (49) that the complete energy spectrum and eigenfunctions for the Hydrogen-like atom given by

$$
\frac{\lambda}{2}=\frac{E_{o s c}^{B}}{4} \Rightarrow\left[E_{a}\right]_{\ell}^{(m)}=\left[E_{a}\right]^{(N)}=-\frac{Z^{2}}{2 N^{2}}, \quad(N=1,2, \ldots)
$$


and

$$
[\psi(\rho, \theta, \varphi)]_{\ell ; \mathrm{j}, m_{j}}^{(m)} \propto \rho^{\ell} \exp \left(-\frac{\rho}{2}\right) L_{m}^{(2 \ell+1)}(\rho)\left\{\begin{array}{l}
y_{+}(\theta, \varphi) \\
y_{-}(\theta, \varphi)
\end{array}\right.
$$

where $E_{\text {osc }}^{B}$ is given by Eq. (44).

Here, $N=\ell+m+1(\ell=0,1,2, \cdots, N-1 ; m=0,1,2, \cdots)$ is the principal quantum

number. Kostelecky and Nieto shown that the supersymmetry in non-relativistic quantum mechanics may be realized in atomic systems [25].

\section{CONCLUSION}

In this work, we have deduced the energy eigenvalues and eigenfunctions of the hydrogen atom via Wigner-Heisenberg (WH) algebra in non-relativistic quantum mechanics. Indeed, from the ladder operators for the 4-dimensional (4D) super Wigner system, ladder operators for the mapped super $3 D$ system, and hence for hydrogen-like atom in bosonic sector, are deduced. The complete spectrum for the hydrogen atom is found with considerable simplicity. Therefore, the solutions of the time-independent Schrödinger equation for the hydrogen atom were mapped onto the super Wigner harmonic oscillator in $4 D$ by using the Kustaanheimo-Stiefel transformation. 


\section{Acknowledgments}

RLR would like to acknowledge CBPF for hospitality. The author also like to acknowledge CES-UFCG of Cuité-PB, Brazil. This research was supported in part by CNPq (Brazilian Research Agency). This work was initiated in collaboration with Jambunatha Jayaraman (In memory), whose advises and encouragement were fundamental.

[1] E. P. Wigner, Phys. Rev. 77, 711 (1950); L. M. Yang, Phys. Rev. 84, 788 (1951); L. O'Raifeartaigh and C. Ryan, Proc. R. Irish Acad. A62, 93 (1963); Y. Ohnuki and S. Kamefuchi, J. Math. Phys. 19, 67 (1978); Y. Ohnuki and S. Watanabe, J. Math. Phys. 33, 3653 (1992); S. M. Plyushchay, Ann. Phys. 245, 339 (1996).

[2] J. Jayaraman and R. de Lima Rodrigues, J. Phys. A: Math. Gen. 23, 3123 (1990).

[3] J. Jayaraman and R. de Lima Rodrigues, Mod. Phys. Lett. A9, 1047 (1994).

[4] S. M. Plyushchay, Int. J. Mod. Phys. A15, 3679 (2000).

[5] S. H. Green, Phys. Rev 90270 (1953); N. Mukunda, E. C. G. Sudarshan, J. K. Sharma and C. L. Mehta, J. Math. Phys. 21, 2386 (1980).

[6] M. A. Vasiliev, Int. J. Mod. Phys. A6, 1115 (1991).

[7] A. P. Polychronakos, Phys. Rev. Lett. 69, 703 (1992).

[8] T. Brzezinski, I. L. Egusquiza and A. J. Macfarlane, Phys. Lett. B311, 202 (1993); L. Brink, T. H. Hansson and M. A. Vasiliev, Phys. Lett. B286, 109 (1992); L. Brink, T. H. Hansson and S. Konstein and M. A. Vasiliev, Nucl. Phys. B401, 591 (1993).

[9] S. Jing, J. Phys. A: Math. Gen. 31, 6347 (1998).

[10] A. Matos-Albiague, J. Phys. A: Math. Gen. 34, 3125 (2001).

[11] R. de Lima Rodrigues, q-deformed Wigner oscillator in quantum mechanics, arXiv:hep-th/0308188 (2003).

[12] T. D. Palev and N. I. Stoilova, J. Phys. A: Math. Gen. 27, 7387 (1994).

[13] R. C. King, T. D. Palev, N. I. Stoilova and J. Van der Jeugt, J. Phys. A: Math. Gen. 36, 4337 (2003), hep-th/0304136.

[14] T. D. Palev, SL(3-N) Wigner quantum oscillators: examples of ferromagnetic-like oscillators with noncommutative, square-commutative geometry, arXiv:hep-th/0601201v2 (2006).

[15] R. C. King, N. I. Stoilova and J. Van der Jeugt, J. Phys. A: Math. Gen. 39, 5763 (2006). 
[16] A. Matos-Albiague, J. Phys. A: Math. Gen. 34, 11059 (2001).

[17] M. A. Lohe and A. Thilagam, J. Phys. A: Math. Gen. 37, 6181 (2004).

[18] M. A. Lohe and A. Thilagam, J. Phys. A: Math. Gen. 38, 461 (2005).

[19] P. Kustaanheimo and E. Stiefel, J. Reine Angew. Math. 218, 204 (1965).

[20] A. C. Chen, Phys. Rev. A22, 333 (1980); Erratum Ibid. A22, 2901 (1980).

[21] F. H. J. Cornish, J. Phys. A: Math. Gen. 17, 323 (1984).

[22] A. C. Chen and M. Kibler, Phys. Rev. A31, 3960 (1985).

[23] E. D'Hoker and L. Vinet, Nucl. Phys. B260, 79 (1985).

[24] V. A. Kostelecky, M. M. Nieto and D. R. Truax, Phys. Rev. D32, 2627 (1985).

[25] V. A. Kostelecky and M. M. Nieto, Phys. Rev. D32, 1293 (1985); Phys. Rev. Lett. 35, 2285 (1984).

[26] R. D. Amado, Phys. Rev. A37, 2277 (1988).

[27] O. L. Lange and R. E. Raab, Operator Methods in Quantum Mechanics, Clarendon Press, Oxford University Press, New York (1991).

[28] R. D. Tangerman and J. A. Tjon, Phys. Rev. A48, 1089 (1993).

[29] F. Cooper, A. Khare and U. Sukhatme, "Supersymmetry in quantum mechanics," World Scientific, Singapure, 2001; B. Bagchi, "Supersymmetry in quantum and classical mechanics," published by Chapman and Hall, Florida (USA), (2000); G. Junker, "Supersymmetric methods in quantum mechanics and statistical physics," Springer, Berlin (1996).

[30] A. Lahiri, P. K. Roy and B. Bagchi, J. Phys. A: Math. Gen. 20, 5403 (1987).

[31] D. Bergmann and Y. Frishman, Y J. Math. Phys. 6, 1855 (1965).

[32] E. Cahill, J. Phys. A: Math. Gen. 23, 1519 (1990).

[33] J. - L. Chen, H. - B. Zhang, X. - H. Wang, and X. - G. Zhao, Int. J. Theor. Phys. 39, 2043 (2000).

[34] G. - J. Zeng, Ka-lin Su and Min Li, Phys. Rev. A50, 4373 (1994).

[35] I. S. Gradshteyn and I. M. Ryzhik, Table of integrals, Series, and Products (4th edition translation edited by A. Jefferey (Academic, New York, 1965), pp. 959.

[36] P. M. Mathews and K. Venkatesan, A text book of quantum mechanics, Tata McGraw-Hill publishing company limited (1978). 